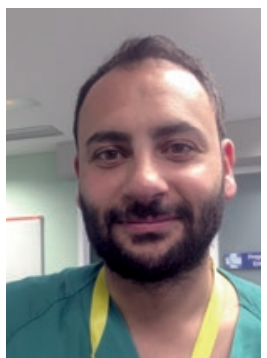

\title{
A debate: MitraClip. Perspectiva del intervencionista
}

\section{Debate: MitraClip. The interventional cardiologist perspective}

\author{
Rodrigo Estévez-Loureiro* \\ Unidad de Cardiología Intervencionista, Servicio de Cardiología, Hospital Universitario Puerta de Hierro-Majadahonda, \\ Majadahonda, Madrid, España
}

VÉASE CONTENIDO RELACIONADO:

https://doi.org/10.24875/RECIC.M19000007

Pregunta: Sin duda, lo más relevante de 2018 en el ámbito de la cardiología intervencionista fueron los tan esperados resultados de los ensayos con MitraClip (Abbott Laboratories, Abbott Park, Illinois, Estados Unidos): el MITRA-FR, en el congreso de la Sociedad Europea de Cardiología (ESC), y el COAPT, en el congreso Transcatheter Cardiovascular Therapeutics (TCT). Se ha comentado mucho sobre los dos ensayos, ¿podría hablarnos de las diferencias básicas que explican los diferentes resultados observados en ellos?

RESPUESTA: Los estudios MITRA-FR ${ }^{1}$ y COAPT $^{2}$ comparten el honor de haber sido los dos primeros estudios de la historia con un diseño riguroso en los que se aleatorizó a pacientes con insuficiencia mitral funcional (IMF) para recibir tratamiento médico óptimo o la combinación de este junto con el dispositivo de corrección de la IMF MitraClip. Como sabemos, los resultados fueron contrapuestos. Mientras que en el MITRA-FR el dispositivo no producía ningún efecto beneficioso sobre el evento compuesto de muerte o rehospitalizaciones al año del seguimiento, en el COAPT el grupo de MitraClip presentó una reducción significativa de las hospitalizaciones a los 2 años, así como una reducción de la mortalidad y del combinado de muerte/rehospitalizaciones a los 2 años. Es lógico analizar las diferencias entre los estudios que puedan justificar dichos hallazgos. A continuación enumero las que considero más relevantes:

- Magnitud de la IMF. Probablemente este es uno de los puntos más destacados que pueden explicar las diferencias. En el estudio MITRA-FR, siguiendo las recomendaciones de las guías de enfermedad de la ESC, se incluyeron pacientes con IMF grave definida como un orificio regurgitante efectivo $(\mathrm{ORE}) \geq 20 \mathrm{~mm}^{2}$ o un volumen de regurgitación $>30 \mathrm{ml}$. Esto hizo que el ORE promedio de los pacientes en este estudio fuese $31 \pm 10 \mathrm{~mm}^{2}$. Por el contrario, en el estudio COAPT, siguiendo las guías americanas ${ }^{3}$ se consideró que la IMF significativa era aquella con ORE $\geq 30 \mathrm{~mm}^{2}$ o con un volumen de regurgitación > $45 \mathrm{ml}$ (ORE promedio: $41 \pm 15 \mathrm{~mm}^{2}$ ). Además, en el MITRA-FR más de la mitad de los pacientes (52\%) presentaba un ORE entre 20 y 30 , mientras que en el COAPT el $87 \%$ de los pacientes tenían valores de ORE $>30 \mathrm{~mm}^{2}$. En resumen, el COAPT incluyó pacientes con IMF más grave que, lógicamente, tendría un mayor impacto en los eventos de los pacientes al ser corregida.

- Volúmenes ventriculares. Los pacientes del estudio MITRA-FR presentaban en promedio unos volúmenes ventriculares más altos que los pacientes del COAPT (volumen telediastólico indexado: $135 \pm 35 \mathrm{ml} / \mathrm{m}^{2}$ frente a $101 \pm 34 \mathrm{ml} / \mathrm{m}^{2}$ ). Esto quiere decir que la enfermedad estaba en una fase más avanzada en el estudio francés que en el americano. Junto con la anterior, puede ser también una de las claves de las diferencias. Tratar poca IMF en pacientes con volúmenes ventriculares muy dilatados puede que no tenga ningún efecto sobre los eventos cardiovasculares mayores.

- Tratamiento médico óptimo. Este también es un punto fundamental para entender las diferencias. En el estudio MITRA-FR, el tratamiento médico que los pacientes recibían antes de la aleatorización fue el que el médico responsable consideraba óptimo. Además, este podía ser modificado a lo largo del seguimiento sin que el comité del estudio pudiese controlar esta variable. Ello puede haber hecho que, a pesar de que las proporciones de fármacos de insuficiencia cardiaca presentadas fuesen las correctas al inicio del estudio, sus dosis pudieron ser modificadas con posterioridad. Y de todos es sabido que la optimización de las dosis de los fármacos tiene un gran impacto en los eventos y en la clase funcional de los pacientes. Este efecto puede verse objetivamente en la gran mejoría en la clase funcional que experimentó el grupo de tratamiento médico del MITRA-FR. Sin embargo, en el COAPT existía un "comité de elegibilidad» que velaba y controlaba que cada paciente estuviese tratado con los fármacos adecuados y a la dosis máxima tolerada antes de la aleatorización. Es por ello que en el seguimiento del COAPT se produjeron muy pocas modificaciones de las dosis respecto a las que había al inicio del estudio.

- Rendimiento de MitraClip. Existen diferencias sustanciales de éxito y rendimiento del dispositivo. En el estudio MITRA-FR, al 9\% de los pacientes ni siquiera se les llegó a implantar el dispositivo. El número de complicaciones fue mayor en el MITRA-FR (14,5\% frente al 8,5\%) y el porcentaje de éxito del

* Autor para correspondencia: Unidad de Cardiología Intervencionista, Hospital Universitario Puerta de Hierro, Manuel de Falla 1, 28220 Majadahonda, Madrid, España. Correo electrónico: roiestevez@hotmail.com (R. Estévez-Loureiro). 
procedimiento al año fue menor en el estudio francés (17 frente al $5 \%$ con IMF $>2+$ ).

- Selección de candidatos. En el estudio COAPT se puso especial cuidado en la selección de los candidatos y se excluyeron aquellos pacientes que, por sus características basales, presentaban un pronóstico malo a corto plazo, y en los que posiblemente la intervención no se acompañase de una mejoría clínica significativa. Entre estas exclusiones estaban la presencia de hipertensión pulmonar grave no reversible, la disfunción del ventrículo derecho moderada o grave, la insuficiencia cardiaca en estadio $\mathrm{D}$, la inestabilidad hemodinámica y el tratamiento inotrópico. Estos pacientes no figuraban en las exclusiones del estudio MITRA-FR. Además, todos los pacientes del MITRA-FR debían haber ingresado al menos una vez en el año previo, mientras que este requisito no era imprescindible en el estudio COAPT, lo que favorece la selección de una población menos evolucionada. La posible inclusión de estos casos junto con la de pacientes con ventrículos en una fase más avanzada de la enfermedad y poca regurgitación mitral puede que sea determinante para explicar sus resultados.

- Tiempo de seguimiento. En el estudio COAPT, las curvas de supervivencia empiezan a separarse claramente alrededor del primer año de seguimiento. El MITRA-FR solo tiene un año de seguimiento. Quizás podamos observar una evolución distinta de los pacientes de este estudio cuando se extienda el seguimiento.

- Aspectos metodológicos. El objetivo primario del estudio MITRA-FR fue el combinado de rehospitalizaciones o muerte al año. Para analizar esta variable se utilizó un análisis de supervivencia de Kaplan-Meier, lo cual es metodológicamente correcto, pero tiene un problema: la rehospitalización es un evento que pude repetirse en el tiempo, y dicho análisis no lo tiene en cuenta. Esto es de especial importancia porque uno de los problemas de este perfil de pacientes es el importante número de reingresos. No es lo mismo reingresar una vez en un año que cinco veces. Este importante matiz no fue captado en el estudio MITRA-FR. Sin embargo, el COAPT sí fue diseñado para analizar los eventos repetidos. De hecho, su objetivo primario es el número de rehospitalizaciones a los 2 años (no solo si se ha ingresado o no). El COAPT incluyó el compuesto de muerte/rehospitalizaciones como un objetivo secundario, pero la forma de analizarlo es distinta a la del MITRA-FR. Los eventos muerte y hospitalización no son excluyentes, aunque sí que uno es más relevante que el otro. En el MITRA-FR, ambos eventos fueron considerados iguales y lo único que cancelaba el análisis de supervivencia era haber padecido uno $u$ otro, el que hubiera sido primero. Sin embargo, el COAPT presenta una forma más apropiada de análisis para este tipo de eventos intercurrentes: la WIN ratio (razón de ganancias sobre pérdidas en el grupo de tratamiento). Esta manera de analizar pondera cuál es evento clínico más importante (la muerte) y le da su relevancia a pesar de que haya ocurrido después de una hospitalización. Esta modalidad de análisis del evento compuesto puede poner de manifiesto diferencias que el método tradicional de Kaplan-Meier no detecta o lo hace en grado menor.

Por último, cabe señalar que el estudio COAPT es más riguroso que el MITRA-FR. En el estudio francés, 43 pacientes fueron excluidos del protocolo en el grupo de MitraClip por diversos motivos, lo cual supone el $28 \%$ de la muestra del grupo de intervención. Además, las numerosas pérdidas de variables secundarias, como calidad de vida, valores analíticos, clase funcional y, lo que es más llamativo, ecocardiogramas de control, hace que estos eventos no hayan sido analizados en el estudio por poder estar sometidos a sesgos. En esta línea, el porcentaje de recidivas de IMF significativa al año es aproximado, lo cual resta exactitud a los análisis.

P.: Se han destacado mucho las virtudes del COAPT y las limitaciones del MITRA-FR, pero ¿cuáles serían para usted los aspectos positivos del MITRA-FR y los aspectos críticos del COAPT?

R.: Los aspectos positivos del MITRA-FR son que es el primer estudio que analiza con cierto rigor este tema y que nos enseña que la elección del tratamiento óptimo para la insuficiencia cardiaca y la optimización hasta las dosis máximas producen una importante mejoría sintomática en los pacientes, a la vez que se reduce la IMF (no olvidemos que esta es un proceso dinámico que puede variar en el tiempo). Además, quizás el aspecto más positivo es que nos señala a qué pacientes probablemente no haya que tratar con esta terapia: pacientes en fase avanzada, con poca regurgitación mitral y sin tener optimizado al máximo el tratamiento. Es posible que debamos evitar este subgrupo de pacientes.

Como aspectos negativos del estudio COAPT señalaría que es un experimento de laboratorio: todas las variables perfectamente controladas, todos los tratamientos al máximo de dosis y un MitraClip con un éxito casi absoluto a los 2 años. Probablemente será difícil replicar esto en la vida real, pero lo que es indiscutible es que la prueba perfecta de que la IMF mata y corregirla puede conllevar una reducción muy importante de los eventos cardiovasculares. El estudio COAPT es el ideal que debemos buscar y al que debemos intentar acercarnos en la vida real.

P.: ¿Cómo se complementan los dos estudios al definir al candidato idóneo para la técnica?

R.: Lo interesante de estos estudios es que pueden tomarse en conjunto para establecer cómo debemos seleccionar a los candidatos. Parece claro que tenemos que evitar a los pacientes con estados avanzados (mayor dilatación ventricular), con IMF no grave y sin estar óptimamente tratados. Si queremos obtener resultados más positivos, nuestros candidatos deben estar en fases más precoces, con un importante grado de IMF (que realmente contribuya a la situación clínica), perfectamente tratados y además tener un muy buen resultado con el dispositivo (por lo que la selección anatómica y la experiencia del equipo implantador deben ser altas). Sin embargo, siempre hay que tomar estas asunciones con cautela. Estos datos nos hablan del paciente "promedio», pero un subanálisis del estudio COAPT nos indica que mientras la IMF sea muy relevante (ORE $>30 \mathrm{~mm}^{2}$ ) siempre se obtiene beneficio, independientemente del grado de dilatación ventricular. Por ello debemos individualizar cada caso $y$, en mi opinión, dar mucha relevancia a la cantidad de IMF. Además de las reducciones de los eventos mayores, debemos tener en cuenta que MitraClip se asocia también con una importante mejoría de la calidad de vida y del grado funcional. También hay que considerar estos objetivos al seleccionar los pacientes, porque a veces esta terapia es lo único que puede servir para paliar síntomas muy avanzados.

P.: En el último TCT vimos también los resultados de un ensayo menor, el REDUCE-FMR, en el que se evaluaba un sistema de anuloplastia percutánea. Dados los resultados con MitraClip, ¿cuál podría ser el papel de los sistemas de anuloplastia percutánea en los pacientes con insuficiencia cardiaca e IMF grave?

R.: En el estudio COAPT, los resultados del clip fueron excelentes porque la selección anatómica de los candidatos fue excelente. Sin embargo, en el mundo real no siempre nos vamos a encontrar esos casos. En mi opinión, en aquellos pacientes que tengan una gran dilatación anular, tanta que incluso obligue a los velos a no coaptar, el clip puede tener resultados subóptimos. Es en estos casos en los que los sistemas de anuloplastia pueden desempeñar un papel fundamental, solos o en combinación con el clip. La idea, y así nos lo 
demuestra el COAPT, es que debemos dejar la menor IMF posible, y la reparación debe ser duradera en el tiempo, porque eso es lo que se va a traducir en una reducción de los eventos cardiovasculares. Los dispositivos de anuloplastia vendrán a complementar el abanico terapéutico para conseguir ese fin. Eso sí, para tenerlos en consideración deben demostrar la misma seguridad y eficacia que MitraClip.

\section{CONFLICTO DE INTERESES}

R. Estévez-Loureiro es proctor de MitraClip y ha recibido una beca de investigación de Abbott Vascular.

\section{BIBLIOGRAFÍA}

1. Obadia JF, Messika-Zeitoun D, Leurent G, et al.; for the MITRA-FR Investigators. Percutaneous Repair or Medical Treatment for Secondary Mitral Regurgitation. N Engl J Med. 2018;379:2297-2306.

2. Stone GW, Lindenfeld J, Abraham WT, et al.; for the COAPT Investigators. Transcatheter Mitral-Valve Repair in Patients with Heart Failure. $N$ Engl J Med. 2018;379:2307-2318.

3. Nishimura RA, Otto CM, Bonow RO, et al. 2017 AHA/ACC Focused Update of the 2014 AHA/ACC Guideline for the Management of Patients With Valvular Heart Disease: A Report of the American College of Cardiology/ American Heart Association Task Force on Clinical Practice Guidelines. Circulation. 2017;135:e1159-e1195. 